\title{
Treinamento de Habilidades Sociais na promoção da autoestima em adolescentes
}

\author{
Social Skills Training in the promotion of self-esteem in adolescents \\ Entrenamiento de Habilidades Sociales para promover la autoestima de adolescentes
}

Recebido: 20/01/2021 | Revisado: 26/01/2021 | Aceito: 29/01/2021 | Publicado: 07/02/2021

\author{
Alessandra da Silva Rodrigues \\ ORCID: https://orcid.org/0000-0003-4317-5217 \\ Universidade Federal de Rondônia, Brasil \\ E-mail: ale.psico@hotmail.com \\ Fabio Biasotto Feitosa \\ ORCID: https://orcid.org/0000-0001-6440-4993 \\ Universidade Federal de Rondônia, Brasil \\ E-mail: fabiobfeitosa@gmail.com \\ Márcia Fortes Wagner \\ ORCID: https://orcid.org/0000-0003-0457-3773 \\ Faculdade Meridional, Brasil \\ E-mail: marcia.wagner@imed.edu.br \\ Reginaldo Pedroso \\ ORCID: https://orcid.org/0000-0001-8987-844X \\ Universidade Federal de Rondônia, Brasil \\ E-mail: pedroso.r9@gmail.com \\ Tomás Daniel Menéndez Rodríguez \\ ORCID: https://orcid.org/0000-0002-4425-1261 \\ Universidade Federal de Rondônia, Brasil \\ E-mail: tomasdanielm@gmail.com \\ Gabrielle Selleri Bezerra \\ ORCID: https://orcid.org/0000-0003-2093-7186 \\ Universidade Federal de Rondônia, Brasil \\ E-mail: gbselleri@gmail.com
}

\begin{abstract}
Resumo
$\mathrm{Na}$ fase transitória da adolescência, a literatura evidencia que a autoestima e as habilidades sociais estão associadas à saúde mental, ao desenvolvimento e à melhor adaptação social. Considerando esta relação, o objetivo da presente pesquisa foi avaliar o efeito de um Treinamento de Habilidades Sociais na promoção da autoestima em adolescentes. Participaram 10 adolescentes, com idades entre 13 e 15 anos, de uma intervenção no desenho pré e pós-teste. Como medida de avaliação foram aplicados na pré e pós-intervenção os instrumentos: Escala de Rosenberg - EAR, Inventário de Habilidades Sociais para Adolescentes - IHSA-Del-Prette, Diário de Campo e Questionários Inicial e Final. Os dados quantitativos foram analisados por meio do software SPSS e os dados qualitativos foram agrupados em categorias temáticas, analisados e utilizados para ilustrar os resultados quantitativos. Os resultados quantitativos e qualitativos apontaram que o Treinamento de Habilidades Sociais foi eficaz no aumento da autoestima em adolescentes, principalmente no aprimoramento das habilidades de Autocontrole e Assertividade. Pode-se concluir que a pesquisa realizada demonstrou que o treinamento de habilidades sociais é um instrumento relevante para elevação da autoestima em adolescentes.
\end{abstract}

Palavras-chave: Adolescência; Treinamento de habilidades sociais; Autoestima.

\begin{abstract}
In the transitional phase of adolescence, the literature shows that self-esteem and social skills are associated with mental health, development and better social adaptation. Considering this relation, the goal of the present research was to evaluate the effect of a Social Skills Training in promoting self-esteem in adolescents. Ten adolescents aged from 13 to 15 took part in an intervention in the pre and post testing scenarios. The assessment measures applied at both pre and post scenarios were Rosenberg Scale, Inventory of Social Skills for Adolescents - IHSA-Del-Prette, Field Notebooks and Initial and Final Questionnaires. The quantitative data were analyzed using the SPSS software and the qualitative data were classified into thematic categories, analyzed and used to illustrate the quantitative results. The quantitative and qualitative results indicated that the Social Skills Training was effective in increasing self-esteem in adolescents, especially in improving self-control and assertive skills. It was concluded that the research realized demonstrated that the Social Skills Training is a relevant instrument for the promotion of self-esteem in adolescents.
\end{abstract}

Keywords: Adolescence; Social skills training; Self-esteem. 


\begin{abstract}
Resumen
En la fase transitoria de la adolescencia, la literatura muestra que la autoestima y las habilidades sociales están asociadas con la salud mental, el desarrollo y a una mejor adaptación social. Teniendo en cuenta esta relación, el objetivo de esta investigación fue evaluar el efecto de una capacitación o Entrenamiento de Habilidades Sociales en la promoción de la autoestima en los adolescentes. Participaron 10 adolescentes, de entre 13 y 15 años, de una intervención en el diseño del tipo pruebas previa y posterior. Como medida de evaluación, se aplicaron los siguientes instrumentos en los instrumentos de pre y post intervención: Escala Rosenberg - EAR, Inventario de Habilidades Sociales para Adolescentes - IHSA-Del-Prette, Diario de Campo y Cuestionarios Inicial y Final. Los datos cuantitativos se analizaron utilizando el software SPSS y los datos cualitativos fueron agrupados en categorías temáticas, analizados y utilizados para ilustrar los resultados cuantitativos. Los resultados cuantitativos y cualitativos indicaron que el entrenamiento en habilidades sociales fue eficaz para aumentar la autoestima en los adolescentes, especialmente en la mejora de las habilidades de autocontrol y de asertividad. Se puede concluir que la investigación demostró que el entrenamiento en habilidades sociales es un instrumento relevante para la elevación de la autoestima en los adolescentes.
\end{abstract}

Palabras clave: Adolescencia; Entrenamiento de habilidades sociales; Autoestima.

\title{
1. Introdução
}

A adolescência é um período de transição da infância para a vida adulta, no qual os adolescentes podem apresentar dificuldades na adaptação familiar, social e escolar devido a diferentes transformações nas relações consigo e com o outro (Moreira, 2018). Nessa etapa, os adolescentes passam significativa parte do tempo na escola, onde vivenciam experiências que podem trazer consequências positivas ou negativas ao bem-estar psicológico. Conflitos interpessoais podem surgir, dificultando a transição (Ferreira et al., 2016) e déficits no repertório de habilidades sociais podem acarretar rejeição pelos colegas, agravando comportamentos depressivos e contribuindo para a baixa autoestima (Campos et al., 2014). abilidades sociais são comportamentos aceitáveis culturalmente, com consequências que maximizam resultados favoráveis para o indivíduo, comunidade e seu grupo social, favorecendo a adaptação social (Del Prette \& Del Prette, 2017).

A aquisição das habilidades sociais ocorre mediante contingências naturais das relações interpessoais na família e grupo social. No entanto, quando o ambiente natural é deficitário ou limitado para o ensino dessas habilidades, estas podem ser construídas por contingências programadas, chamadas de Treinamento de Habilidades Sociais (THS), que consistem em estratégias e técnicas de intervenções para aprimorar e ampliar o repertório de habilidades sociais (Del Prette \& Del Prette, 2017).

Pesquisas com diferentes públicos evidenciam a importância das habilidades sociais na promoção da saúde. Conforme a análise de Feitosa (2014), aprimorar as habilidades sociais possibilita a alteração do contexto vivencial no sentido de ampliar os reforçadores sociais, contribuindo significativamente para diminuir o estresse e os sintomas depressivos, bem como melhorando a autoconfiança e autoestima. O termo autoestima representa um aspecto de autoavaliação, englobando pensamentos e sentimentos de orientação positiva (autoaprovação) ou negativa (depreciação), incluindo a percepção de autovalor, associados ao autoconceito, ao que a pessoa faz e à sua relação com o grupo social (Hutz \& Zanon, 2011; Schultheisz \& Aprile, 2015). Na Análise do Comportamento, a autoestima é definida como um sentimento adquirido através das contingências de reforçamento positivo de origem social (Meyer, 2011).

Assim como as habilidades sociais, a autoestima frequentemente está associada a diferentes indicadores de saúde mental, realização acadêmica e adaptação social (Hutz \& Zanon, 2011; Paixão et al., 2018). A autoestima na adolescência é considerada a variável mais crítica para o estabelecimento das relações sociais desse grupo, influenciando seu engajamento em projetos, isto é, adolescentes com bons níveis de autoestima tendem a ser mais persistentes e, consequentemente, obter mais progressos frente aos com baixos níveis para uma mesma tarefa difícil (Castro Sena \& Maia, 2017).

Neste contexto, quando a saúde mental do adolescente apresenta prejuízos, podem ocorrer comportamentos autolesivos e, entre eles, o suicídio. Sabe-se que o suicídio é crescente em todo o mundo e que a ideação suicida ocorre dentro de um quadro que inclui baixa autoestima, conflitos interpessoais e sintomas depressivos (Sábado et al., 2020). Apesar desse 
entendimento, a literatura especializada carece de trabalhos mostrando relações estáveis e diretas entre habilidades sociais e autoestima, deixando de responder se o THS seria um recurso técnico viável para elevar a autoestima de adolescentes, uma vez que poderia favorecer sua adaptação social.

Sabe-se que o repertório comportamental restrito de autocontrole tende a acompanhar dificuldade no manejo de situações aversivas (Fogaça et al., 2019). Enquanto que as habilidades assertivas, como saber negociar interesses conflitantes, podem facilitar o relacionamento interpessoal (Leme et al., 2015), consequentemente, afetariam a autoestima.

No entanto, a literatura internacional indica dados inconclusivos sobre a capacidade do THS em elevar a autoestima de adolescentes. Conforme Morowatisharifabad et al. (2019), ao ser realizado um estudo transversal correlacional com 364 adolescentes iranianas na faixa etária de 12 a 14 anos, foi possível identificar a associação entre habilidades para a vida e autoestima. Os autores defenderam a viabilidade de programas educativos impactarem na autoestima e na saúde mental de adolescentes, como seria o caso de um programa de habilidades sociais. Contudo, em outro estudo com 109 adolescentes portugueses, Mota e Matos (2013) não encontraram relações significativas entre habilidades sociais e autoestima. Mas defenderam que elevados níveis de comunicação e confiança nos pares podem gerar segurança entre adolescentes e elevar sua autoestima, à medida que sentem maior capacidade para expressar suas ideias e sentimentos, compartilhar dificuldades e aprimorar estratégias para enfrentá-las. Por sua vez, Seema e Kumar (2018) realizaram uma intervenção de THS com 200 adolescentes iranianos em doze sessões, tendo encontrado redução nas agressões verbais; porém, não houve diferenças significativas entre o grupo controle e o grupo experimental na avaliação da autoestima, diferentemente do esperado.

Nesse sentido, os poucos estudos internacionais encontrados apresentam a hipótese que habilidades sociais elevam a autoestima de adolescentes; porém, sem ainda haver pesquisas suficientes que a sustente. No Brasil, não foi identificada nenhuma publicação de pesquisa de intervenção com o THS testando essa hipótese. Apesar desta lacuna, pesquisas no contexto nacional têm demonstrado a relevância das habilidades sociais na promoção da qualidade das relações interpessoais de adolescentes (Cardoso et al., 2018; Leme et al., 2016), bem como no seu desempenho acadêmico (Feitosa et al., 2012; Jovarini et al., 2018). Nessa direção, Coelho e Dell’Aglio (2019, p. 250) apontam que a escola deve ser considerada “[...] um cenáriochave para intervenções destinadas à promoção do bem-estar dos alunos”. Reconhece-se que a escola, além de ter seu objetivo pautado na formação acadêmica, exerce influência sobre o comportamento humano, uma vez que desenvolve a aprendizagem, os comportamentos, os valores e as habilidades sociais (Ferreira et al., 2016).

Considerando a participação das habilidades sociais e da autoestima na saúde, desenvolvimento e adaptação social de adolescentes, o objetivo da presente pesquisa foi avaliar o efeito de um Treinamento de Habilidades Sociais na promoção da autoestima em adolescentes.

\section{Método}

A pesquisa realizada foi baseada no método quantitativo, no qual, segundo Pereira et al. (2018), faz-se a coleta de dados quantitativos que podem ser analisados estatisticamente, como no caso do presente estudo. No entanto, além da análise de dados quantitativos, também foram utilizados questionários abertos e diário de campo, os quais geraram dados qualitativos que também foram analisados e utilizados como dados adicionais, para ilustrar os resultados quantitativos. Todos os instrumentos utilizados são explicitados nesta seção.

Foi feita uma intervenção com amostra não clínica, com desenho pré e pós-teste. Participaram deste estudo 10 adolescentes (oito meninas e dois meninos) com idades entre 13 e 15 anos $(M=14,20 ; d p=0,6)$, estudantes do $9^{\circ}$ ano do Ensino Fundamental de uma escola privada. Os dados quantitativos, que foram, posteriormente, analisados estatisticamente, foram coletados com o uso de dois instrumentos de avaliação: a Escala de Rosenberg - EAR e o Inventário de Habilidades 
Sociais para Adolescentes - IHSA-Del-Prette. Os dados qualitativos adicionais foram coletados com o uso de um questionário inicial, um questionário final e diário de campo.

A Escala de Rosenberg - EAR, cuja versão utilizada foi a adaptada e validada por Hutz (2000) por apresentar consistência interna satisfatória (alfa de Cronbach=0,90) e confiabilidade em pesquisas brasileiras (Hutz \& Zanon, 2011; Sbicigo et al., 2010). A EAR é composta por dez afirmações relacionadas a um conjunto de sentimentos de autoestima e autoaceitação e a classificação dos níveis de autoestima para faixa etária de 13 a 15 anos, de ambos os sexos são (Hutz \& Zanon, 2011): baixo (percentis de 05 a 25); médio inferior (35 a 50); médio superior (60 a 75) e alto (80 a 95).

O Inventário de Habilidades Sociais para Adolescentes - IHSA-Del-Prette trata-se de um instrumento de autorrelato que avalia habilidades sociais e situações interpessoais em dois indicadores: frequência e dificuldade (no presente estudo foi utilizada somente a dimensão frequência). É composto por 38 questões que incluem diferentes habilidades divididas em seis fatores: F1-Empatia (Expressar compreensão e apoio, elogiar, fazer amizades, pedir desculpas, preocupar-se com o bem-estar do outro etc.); F2-Autocontrole (Reúne as habilidades de reagir com calma a situações aversivas em geral); F3-Civilidade (Agradecer favores, cumprimentar, elogiar, fazer pequenas gentilezas); F4-Assertividade (Recusar pedidos abusivos, demonstrar desagrado, encerrar conversas, conversar com pessoas de autoridade etc.); F5-Abordagem Afetiva (Estabelecer contato e conversação para relações de amizade, unir-se a grupos, expressar satisfação e insatisfação a diferentes formas de carinhos etc.) e F6-Desenvoltura Social (Pedir informações, explicar tarefas aos colegas, apresentação de trabalhos em grupos etc.). As respostas são organizadas em uma escala Likert que varia de zero (nunca) a 04 (sempre), classificando-se em cinco posições (abaixo da média inferior; médio inferior; dentro da média; elaborado e altamente elaborado). Conforme Del Prette e Del Prette (2015), este é um instrumento validado na avaliação de habilidades sociais em adolescentes e por apresentar bons índices de consistência interna ( $\alpha=0,89$ para a escala total e 0,68 a 0,85 para fatores de frequência).

Além dos instrumentos citados, foram aplicados dois questionários. O Questionário Inicial, elaborado para esse estudo, é composto por sete perguntas abertas com o objetivo de identificar as habilidades deficitárias e a maneira como os adolescentes se percebiam. Esse instrumento, apesar de não ser analisado em sua totalidade no presente estudo, serviu de base para o planejamento das atividades e técnicas propostas na intervenção, além de fornecer dados adicionais para ilustrar os resultados quantitativos. As perguntas contidas são: “Em sua opinião, quais são suas principais qualidades?”; “Em sua opinião quais são seus principais defeitos?”; “O que você gostaria de mudar em você?”; “Como você avalia seu relacionamento com a família?"; "Como você avalia sua relação com amigos e professores?"; "Você está satisfeito de viver? Por quê?”; e "Você acha que tem um bom nível de autoestima?".

O Questionário Final continha as mesmas perguntas compostas no questionário inicial, acrescido das seguintes: “Como você se sentiu durante as sessões do treinamento?”; "O treinamento foi útil para você? Por quê?"; "Nos dois últimos meses, tem percebido mudanças em você? Se sim, descreva!"; "Se você pudesse deixar uma sugestão a outras pessoas com baixa autoestima, o que seria?"; e “Gostaria de deixar algum comentário ou sugestão sobre o treinamento?". Este instrumento foi baseado no trabalho de Pereira-Guizzo et al., (2018), em que sugerem entrevistas de relatos para avaliação do processo e os efeitos da intervenção, a fim de acompanhar a manutenção do aprendizado e a efetividade.

Por fim, foi utilizado também um Diário de campo para descrever, analisar e registrar os acontecimentos relevantes durante cada sessão do treinamento (assiduidade/frequência, execução das atividades, relatos e progressos pessoais), servindo também para registrar respostas e evolução dos adolescentes durante a intervenção.

Quanto às considerações éticas, este estudo seguiu os preceitos básicos recomendados pela Resolução $n^{\circ} 466 / 12$ do Conselho Nacional de Saúde em relação às Diretrizes e Normas Regulamentadoras de Pesquisas Envolvendo Seres Humanos. Primeiramente, foi realizada a apresentação do projeto de pesquisa à instituição em que foi realizado o presente estudo e, 
mediante autorização e assinatura da Carta de Anuência, o projeto foi submetido e aprovado pelo Comitê de Ética da Universidade Federal de Rondônia sob o CAAE de número 11254419.2.0000.5300.

Após a aprovação pelo comitê, em conjunto com a psicóloga da instituição, foi feita a pré-seleção da turma que participou do presente estudo. A turma selecionada foi a do nono ano por apresentar, segundo o corpo docente, muitas demandas interpessoais. Logo após, em sala de aula, foi realizado o convite aos alunos, onde foram comunicados que dez pessoas participariam da pesquisa e que haveria sorteio ou uso de decisões técnicas para a seleção final dos alunos que se enquadrassem no critério de inclusão (baixa autoestima). Os responsáveis legais foram contatados por telefone e convidados a participar de uma reunião cujo objetivo foi falar sobre a importância da autoestima e das habilidades sociais na adolescência, bem como, apresentar os objetivos e os procedimentos da pesquisa. Por não obter êxito em todas as ligações, foram entregues bilhetes aos alunos com as informações da reunião, ressaltando que a participação era voluntária e confirmada mediante a assinatura dos adolescentes e dos responsáveis legais nos Termo de Assentimento Livre e Esclarecido (TALE) e Termo de Consentimento Livre e Esclarecido (TCLE), respectivamente. Após a reunião com os responsáveis e a obtenção das assinaturas dos termos legais, foi realizado o pré-experimento com avaliação pré e pós-intervenção, que se deu em três momentos.

$1^{\circ}$ Momento: Ocorreu antes da intervenção e foi dividido em três etapas, sendo a primeira etapa, a aplicação em sala de aula da Escala de Autoestima de Rosenberg- EAR com todos os alunos da turma pré-selecionada. Como critério de inclusão, participaram da pesquisa alunos que apresentaram níveis baixos de autoestima (percentis entre 05 e 25). Dos vinte e quatro alunos que responderam a escala, dezoito se enquadraram; desta forma, foram selecionados os dez que obtiveram os menores níveis. Na segunda etapa, ocorreu a aplicação coletiva do IHSA-Del-Prette com estes dez participantes. Por fim, na terceira etapa, realizou-se uma entrevista individual, cujo objetivo foi a construção do vínculo com cada participante, o levantamento de dados sociodemográficos, bem como, o esclarecimento de dúvidas acerca do treinamento. Nesta etapa foi aplicado individualmente o Questionário Inicial.

$2^{\circ}$ Momento: Realização da intervenção, que ocorreu nas dependências da instituição e foi concretizada por meio de dez sessões grupais com duração de 50 minutos cada, sendo um encontro por semana. Para o melhor desempenho do grupo, contou-se com a participação voluntária de uma coterapeuta (estudante do oitavo período de psicologia, que foi treinada previamente e instruída sobre os procedimentos do treinamento), a qual, por intermédio do diário de campo, registrou e descreveu os acontecimentos relevantes durante cada sessão. Todas as etapas do treinamento foram realizadas em horário de aula, em comum acordo com todos os alunos, seus responsáveis e equipe pedagógica.

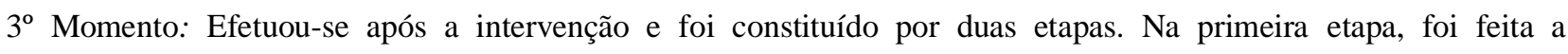
reaplicação coletiva da EAR e do IHSA-Del-Prette. Na segunda etapa, foi feita a aplicação individual do Questionário Final.

Quanto ao Treinamento de Habilidades Sociais, este se deu de forma gradativa: Nas primeiras sessões foram treinadas as habilidades sociais básicas que incluem: observar e descrever os desempenhos sociais (passivo, assertivo, agressivo), identificar emoções, realizar feedbacks etc. Progressivamente, foram trabalhadas as habilidades intermediárias que tiveram foco nas queixas dos participantes relatadas durante as sessões e avaliadas pelo IHSA-Del-Prette na pré-intervenção (como, cumprimentar, apresentar-se a estranhos, fazer e receber elogios). E por fim, habilidades como, ser assertivo, saber lidar com críticas, expor sentimentos positivos e negativos, demonstrar desagrado, etc.

Esta metodologia foi seguida conforme o padrão de progressão dos objetivos em sessões de Programas de Treinamento de Habilidades Sociais sugeridos por Del Prette e Del Prette (2017). Na Tabela 1, encontram-se as habilidades sociais focadas em cada sessão. 
Tabela 1. Habilidades Sociais trabalhadas em cada sessão do THS.

\begin{tabular}{|c|c|c|}
\hline Sessão & Temas & Habilidades Sociais \\
\hline $1^{\mathrm{a}}$ & Integrar para conhecer & $\begin{array}{l}\text { Integração dos participantes; Contrato terapêutico; Entendimento sobre } \\
\text { o desempenho social (passivo, assertivo e agressivo). }\end{array}$ \\
\hline $2^{\mathrm{a}}$ & $\begin{array}{l}\text { Identificando as } \\
\text { emoções: Sentir, } \\
\text { valorizar e aprimorar. }\end{array}$ & $\begin{array}{l}\text { Reconhecer e nomear as emoções; Promover valorização dos } \\
\text { sentimentos pessoais e de outras pessoas; Geração de alternativas para } \\
\text { lidar com os sentimentos. }\end{array}$ \\
\hline $3^{\mathrm{a}}$ & Empatia: O que é? & Identificar sentimentos e problemas dos outros; Expressar \\
\hline & Como usá-la? & $\begin{array}{l}\text { solidariedade e apoio diante às emoções e dificuldades do outro; } \\
\text { Oferecer ajuda; }\end{array}$ \\
\hline $4^{\mathrm{a}}$ & Falo ou não falo? & $\begin{array}{l}\text { Apresentar-se a estranhos (cumprimentar, elogiar etc.); Identificar } \\
\text { grupos abertos e fechados; Inserir-se em grupos, realizando perguntas } \\
\text { para fazer amizades. }\end{array}$ \\
\hline $5^{\mathrm{a}}$ & Elogiar e ser elogiado & Fazer e receber elogios. \\
\hline $6^{\mathrm{a}}$ & Quero? Posso? Devo? & $\begin{array}{l}\text { Realizar e negar pedidos; Conversar com pessoas de autoridade; } \\
\text { Resistir à pressão de grupos; Demonstrar desagrado. }\end{array}$ \\
\hline $7^{\mathrm{a}}$ & $\begin{array}{l}\text { Reconhecer para } \\
\text { crescer }\end{array}$ & $\begin{array}{l}\text { Identificar críticas construtivas e destrutivas; Lidar com críticas } \\
\text { mantendo o autocontrole; Aprender a dar Feedbacks. } \\
\text { Expressar sentimentos positivos e negativos; Identificar problemas; }\end{array}$ \\
\hline $8^{\mathrm{a}}$ & Falar ou calar? & $\begin{array}{l}\text { Formular gerações de alternativas; Escolher estratégia de } \\
\text { enfrentamento. }\end{array}$ \\
\hline $9^{a}$ & $\begin{array}{l}\text { Eu seria mais feliz } \\
\text { se... }\end{array}$ & $\begin{array}{l}\text { Expressar sentimentos positivos e negativos; Reconhecer habilidades } \\
\text { pessoais; Reconhecimento do problema; Implementações de soluções. } \\
\text { Descricão do que aprenderam e o que mais gostaram do THS; Tirando }\end{array}$ \\
\hline $10^{\mathrm{a}}$ & Eu aprendi que... & $\begin{array}{l}\text { as dúvidas emergentes sobre os comportamentos socialmente } \\
\text { habilidosos; Feedbacks e encerramento do grupo. }\end{array}$ \\
\hline
\end{tabular}

Fonte: Autores.

As sessões foram planejadas de acordo com as habilidades sociais mais deficitárias dos participantes, conforme tabulações no pré-teste e respostas obtidas através do Questionário Inicial. As técnicas utilizadas durante os encontros foram: vivências, modelagem, modelação, desempenho de papéis (role-play), ensaio comportamental, psicoeducação, tarefas interpessoais de casa (TIC), feedback, etc.

As análises de dados, feitas para o grupo amostral dos participantes, basearam-se nos resultados da EAR e dos indicadores de "Frequência" do IHSA-Del-Prette analisados por meio do Statistical Package for the Social Sciences for Windows (SPSS, versão 22.0). O Wilcoxon ${ }^{@}$ foi utilizado para comparar os resultados da pré e pós-intervenção de ambos os instrumentos. $\mathrm{O}$ índice de significância utilizado foi de 0,05. As anotações do diário de campo bem como as respostas dos Questionários Inicial e Final foram analisadas e agrupadas em categorias temáticas, sendo algumas delas utilizadas no presente estudo para ilustrar os resultados positivos alcançados e confirmados pela análise estatística. Para descrição dos relatos, os participantes foram nomeados como, $p 1, p 2, p 3 \ldots p 10$.

\section{Resultados}

A participação dos adolescentes no treinamento foi satisfatória, não ocorrendo desistências. Os integrantes comportaram-se de forma assídua e, nos encontros que tiveram faltas, todas foram justificadas. Em relação à frequência dos participantes, $10 \%(n=1)$ estiveram presentes em oito sessões, $20 \%(n=2)$ participaram de nove sessões e $70 \%(n=7)$ participaram de todas as sessões. Esse fator contribuiu para o bom andamento do grupo, pois, além de fortalecer o vínculo terapêutico, viabilizou a realização das atividades propostas.

Os resultados apontaram diferenças estatisticamente significativas no escore total e em alguns dos fatores do IHSADel-Prette. A Tabela 2 apresenta a análise da Frequência do Escore Total e dos Fatores das Habilidades Sociais na pré e pós- 
intervenção da amostra grupal dos dez participantes. Verificou-se por meio do teste Wilcoxon que houve aumento no pós-teste no Escore Total $(z=-1,99, p=0,047)$ e nos fatores F2-Autocontrole $(z=-2,45, p=0,015)$ e F4-Assertividade $(z=-1,96, p=0,049)$.

Tabela 2. Análise de Comparação da Frequência do Escore Total e dos Fatores das Habilidades Sociais na Pré e PósIntervenção.

\begin{tabular}{lllc}
\hline \multirow{2}{*}{\multicolumn{1}{c}{ Indicador }} & \multicolumn{2}{c}{ PRÉ-INTERVENÇÃO } & \multicolumn{2}{c}{ PÓS-INTERVENÇÃO } \\
\cline { 2 - 4 } & \multicolumn{1}{c}{$\overline{\mathrm{X}}(\mathrm{DP})$} & $\overline{\mathrm{X}}(\mathrm{DP})$ & Diferença \\
\hline Escore Total & $66,6(23,27)$ & $90,1(32,63)$ & PRÉ<PÓS* \\
F1-Empatia & $23,8(7,67)$ & $27,8(8,51)$ & - \\
F2-Autocontrole & $9,9(7,16)$ & $16,8(7,61)$ & PRÉ<PÓS* \\
F3-Civilidade & $15,4(4,53)$ & $16,8(5,63)$ & - \\
F4-Assertividade & $11,6(7,07)$ & $16,6(8,42)$ & PRÉ<PÓS* \\
F5-Abordagem Afetiva & $6(4,05)$ & $10,5(4,22)$ & - \\
F6-Desenvoltura Social & $8,3(4,45)$ & $11,9(5,11)$ & - \\
\hline
\end{tabular}

Nota PRÉ = Pré-teste; PÓS = Pós-teste; $\bar{X}=$ Média; DP = Desvio Padrão; - = Resultados sem diferenças estatísticas significativas. $*_{p}<0,05$.

Fonte: Autores.

Referente ao Escore Total, conforme a Tabela 3, no pré-teste, 70\% da amostra apresentava repertório comportamental Abaixo da Média Inferior ( $n=7), 10 \%$ possuía repertório considerado Médio Inferior ( $\mathrm{n}=1)$ e apenas $20 \%$ estava Dentro da Média ( $\mathrm{n}=2)$. Nenhum participante obteve repertório comportamental considerado Elaborado e/ou Altamente Elaborado. No pós-testes, $57 \%(\mathrm{n}=4)$ da amostra deixou de apresentar repertório Abaixo da Média Inferior, $10 \%$ obteve repertório Médio Inferior $(n=1), 20 \%$ esteve Dentro da Média $(n=2), 10 \%$ passou a apresentar repertório Elaborado (n=1) e 30\% obteve repertório Altamente Elaborado $(n=3)$.

Tabela 3. Análise do Escore Total das Habilidades Sociais Pré e pós-intervenção.

\begin{tabular}{lclll}
\hline \multirow{2}{*}{ Participantes } & \multicolumn{3}{c}{ Pré-intervenção } & \multicolumn{1}{c}{ Pós-intervenção } \\
\cline { 2 - 5 } & ET (P) & \multicolumn{1}{c}{ C.G } & ET (P) & \multicolumn{1}{c}{ C.G } \\
\hline$p 1$ & $88,00(30)$ & Médio Inferior & $94,00(40)$ & Dentro da Média \\
$p 2$ & $68,00(15)$ & Abaixo da Média Inferior & $103,00(70)$ & Elaborado \\
$p 4$ & $56,10(5)$ & Abaixo da Média Inferior & $50,00(3)$ & Abaixo da Média Inferior \\
$p 5$ & $36,27(1)$ & Abaixo da Média Inferior & $36,27(1)$ & Abaixo da Média Inferior \\
$p 6$ & $68,00(10)$ & Abaixo da Média Inferior & $88,00(30)$ & Médio Inferior \\
$p 7$ & $50,00(3)$ & Abaixo da Média Inferior & $128,90(95)$ & Altamente Elaborado \\
$p 8$ & $94,00(40)$ & Dentro da Média & $128,90(95)$ & Altamente Elaborado \\
$p 9$ & $55,58(3)$ & Abaixo da Média Inferior & $120,00(85)$ & Altamente Elaborado \\
$p 10$ & $84,58(20)$ & Abaixo da Média Inferior & $63,90(5)$ & Abaixo da Média Inferior \\
\hline
\end{tabular}

Nota . ET= Escore Total; P=Percentil; $\mathrm{CG}=$ Classificação Geral. Fonte: Autores. 
Considerando o desempenho individual nas seis subescalas de habilidades sociais, antes e depois da intervenção, foi possível observar os seguintes efeitos: em relação à F1-Empatia, no pré-teste nenhum participante obteve repertório comportamental considerado Elaborado e Altamente Elaborado. No pós-teste, $20 \%(\mathrm{n}=2)$ da amostra passou a apresentar tais resultados (Elaborado 10\%, e Altamente Elaborado 10\%).

Referente ao fator F2-Autocontrole, $80 \%(\mathrm{n}=8)$ da amostra apresentava repertório Abaixo da Média Inferior e Média Inferior no pré-teste. No pós-teste, este resultado caiu para $40 \%(n=4)$ e $60 \%(n=6)$ da amostra geral que apresentou resultados favoráveis (Dentro da Média 10\%, n=1; Elaborado 20\%, n=2; e Altamente Elaborado 30\%, n=3).

Quanto à F3-Civilidade, 70\% $(\mathrm{n}=7)$ dos participantes apresentavam repertório Abaixo da Média Inferior e Média Inferior. No pós-teste, este dado caiu para $40 \%(n=4)$, e a amostra passou a apresentar repertório Dentro da Média (40\%, $n=4)$, Elaborado $(10 \%, \mathrm{n}=1)$ e Altamente Elaborado $(10 \%, \mathrm{n}=1)$.

Na subescala F4-Assertividade foi evidenciado que, no Pré-teste, $80 \%(n=8)$ da amostra apresentava repertório Abaixo da Média Inferior e, no pós-teste, este dado foi reduzido para 40\% (n=4). Os outros 60\% (n=6) passou a apresentar repertórios Dentro da Média (20\%, n=2), Elaborado (20\%, n=2) e Altamente Elaborado (20\%, n=2).

Na categoria F5-Abordagem Afetiva, $80 \%(\mathrm{n}=8)$ da amostra apresentava repertório Abaixo da Média Inferior antes da intervenção. Após o treinamento, este dado caiu para $50 \%(\mathrm{n}=5)$ e os outros $50 \%(\mathrm{n}=5)$ da amostra geral passou a apresentar resultados Dentro da Média (30\%, n=3), Elaborado (10\%, n=1) e Altamente Elaborado (10\%, n=1).

Por fim, sobre a F6-Desenvoltura Social, $60 \%(n=6)$ dos adolescentes apresentavam repertórios Abaixo da Média Inferior no pré-teste, destes, 30\% (n=3) melhoraram sua classificação no pós-teste. Na classificação pós-teste, 70\% (n=7) da amostra passou a apresentar resultados Dentro da Média (30\%, n=3), Elaborado (20\%, n=2) e Altamente Elaborado (20\%, $\mathrm{n}=2)$.

Quanto aos resultados da EAR, foram encontradas diferenças estatisticamente significativas nos escores pré e pósintervenção. O aprimoramento do repertório comportamental influenciou positivamente no aumento da autoestima global de todos participantes $(z=-2,80, p=0,005)$, como pode ser visto na Tabela 4.

Tabela 4. Análise de Comparação do Escore Total e Percentil da Autoestima Pré e Pós-Intervenção.

\begin{tabular}{llcr}
\hline & \multicolumn{2}{c}{ PRÉ } & \multicolumn{2}{c}{ PÓS } \\
\cline { 2 - 4 } & \multicolumn{1}{c}{$\overline{\mathrm{X}}(\mathrm{DP})$} & $\overline{\mathrm{X}}(\mathrm{DP})$ & Sig. \\
\hline Autoestima Escore Total & $16,70(2,627)$ & $29,70(3,743)$ & 0,005 \\
Autoestima Percentil & 5,00 & 55,00 & \\
\hline
\end{tabular}

Nota. PRÉ=Pré-teste; PÓS=Pós-teste; $\bar{X}=$ Média; DP=Desvio Padrão; Sig.=Significância $p<0,05$. Fonte: Autores.

Referente aos níveis de Classificação Geral de autoestima (baixo, médio inferior, médio superior e alto), foi evidenciado no pré-teste que todos os participantes apresentavam níveis baixos de autoestima. No pós-teste, 70\% (n=7) da amostra passou a apresentar melhores classificações. Destes, 10\% $(\mathrm{n}=1)$ aumentou seu nível de baixo para médio inferior, 30\% $(n=3)$ passou de baixo para médio superior, e 30\% $(n=3)$ alcançou níveis altos de autoestima, conforme estão apresentados na Tabela 5 .

Embora 30\% (n=3) dos participantes não tenha mudado a classificação geral de sua autoestima, permanecendo nos níveis baixos, elevação nos escores totais e nos percentis foi evidenciada. Destes, um adolescente ( $p 9)$ elevou seu percentil de 05 para $10 \%$ e dois participantes ( $p 7$ e p 10) aumentaram de 05 para $25 \%$. Portanto, no que se refere ao desenvolvimento da autoestima, evidenciam-se diferenças estatisticamente significativas na pós-intervenção com todos os participantes, ilustradas pela Figura 1. 
Figura 1. Gráfico indicativo das variações dos valores médios de autoestima.

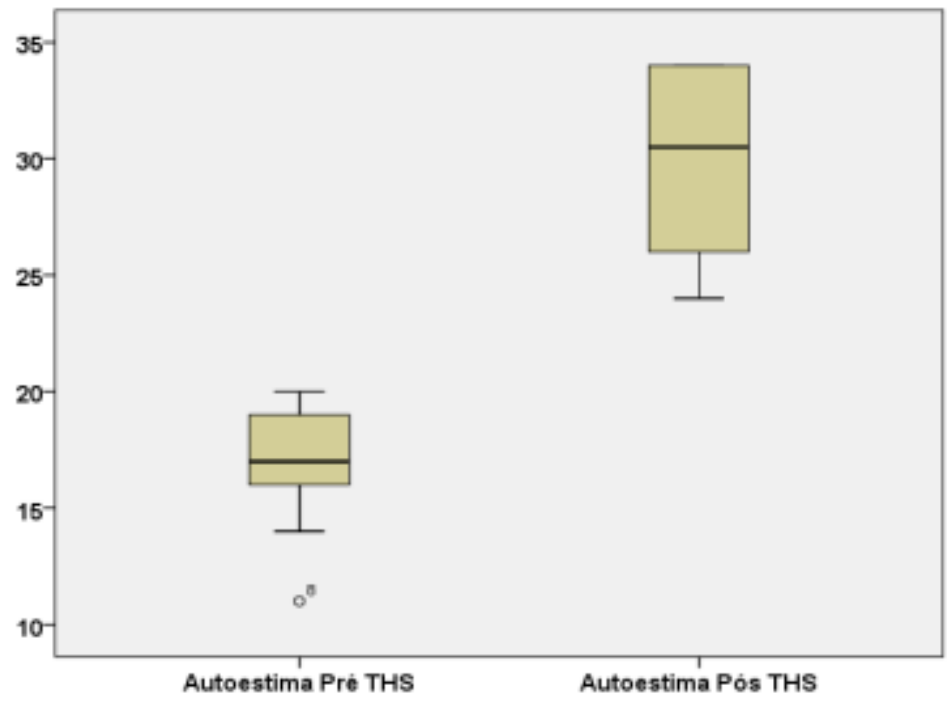

Fonte: Autores.

As anotações no Diário de Campo, bem como as descrições obtidas no Questionário Final, contribuíram com esses resultados estatisticamente significativos e fortaleceram a importância desse tipo de programa para adolescentes: "Eu sentia muito medo de esclarecer o que eu sinto e agora eu percebo que não é preciso isso. Passei a gostar um pouco mais de mim." ( $p 7)$; “Comecei a agir com mais maturidade e vi que tinham muitas coisas simples de serem mudadas." (p3); "O Treinamento me ajudou ser mais empática, a me enturmar mais com as pessoas (mesmo eu ainda tendo um pouco de dificuldade), mas melhorei bastante comparado a antes." $(p 9)$; "Me ajudou mais do que eu esperava, foi incrível passar um tempo aprendendo coisas novas, principalmente como lidar com os problemas e desafios." ( $p 8)$; "Acho que estou conseguindo demonstrar mais quando algo me incomoda, a minha autoestima fica elevada com mais frequência." ( $p 10)$.

\section{Discussão}

No presente estudo, foi possível identificar que o Treinamento de Habilidades Sociais, fundamentado no padrão de progressão sugerido por Del Prette e Del Prette (2017) e utilizando estratégias terapêuticas com procedimentos comportamentais e cognitivo-comportamentais, foi eficaz para o aprimoramento das habilidades sociais refletindo na promoção da autoestima de adolescentes em ambiente escolar. Assim, divergindo de estudos prévios (Mota \& Matos, 2013; Seema \& Kumar, 2018), e corroborando o estudo de Morowatisharifabad et al. (2019), no presente estudo confirma-se a hipótese de que habilidades sociais são capazes de impactar na autoestima de adolescentes, reduzindo a lacuna existente na literatura sobre o tema e com o público pesquisado.

Quanto à autoestima, o treinamento de habilidades sociais empreendido demonstrou ser efetivo, apresentando melhores médias na Escala de Rosenberg para todos os participantes após intervenção. Por meio da análise das afirmativas presentes na EAR, constatou-se que no pré-teste, $100 \%(\mathrm{n}=10)$ dos participantes consideravam-se "inúteis" e não se sentiam pessoas de valor, $90 \%(n=9)$ não estavam satisfeitos consigo, 80\% $(n=8)$ se viam como fracassados, sem boas qualidades e acreditavam não ter motivos para se orgulhar. No pós-teste, $100 \%(\mathrm{n}=10)$ passou a encontrar motivos que os fizessem sentir orgulho de si, desconstruindo a ideia de serem pessoas fracassadas, $90 \%(n=9)$ passou a se considerar como pessoa de valor e com boas qualidades pessoais e $80 \%(n=8)$ sentia satisfação pessoal.

Em relação às habilidades sociais, no pós-teste verificou-se aumento estatisticamente significativo na frequência das habilidades sociais gerais, revelando a eficácia do THS no fim a que se destina, sobretudo, para o Autocontrole e a 
Assertividade. Resultados que se destacam, visto que pessoas com repertório comportamental restrito de autocontrole apresentam baixa tolerância à frustração, impulsividade e possuem dificuldade no manejo de situações aversivas (Fogaça et al., 2019). E as habilidades assertivas são relevantes para o bem-estar dos adolescentes, pois aprender habilidades como expressar sentimentos negativos, solicitar mudanças de comportamentos e saber negociar interesses conflitantes podem facilitar o relacionamento interpessoal (Leme et al., 2015), consequentemente impactando na autoestima.

Conforme expõem Sábado et al. (2020), adolescentes com baixa autoestima apresentam tendência a impulsividade, insegurança, medo exagerado de errar e dificuldade para tomar decisões, requerendo intervenção em saúde. Desse modo, no THS realizado, quanto ao Autocontrole, foram trabalhadas as estratégias para reagir com calma diante das situações aversivas, apontando alternativas possíveis para expressar seus sentimentos, respeitando seus direitos de forma socialmente competente, sem ofensas ou revide.

Sobre a Assertividade, à medida que as sessões foram ocorrendo através de um ambiente acolhedor e sem julgamentos, foi perceptível o quanto os participantes sentiam-se mais confiantes para realizar as atividades, aumentando a participação nos debates. Aumentou também a expressão de sentimentos positivos e negativos, a defesa de seus direitos e a descrição de suas qualidades pessoais.

Quanto às demais subescalas do IHSA-Del-Prette (F1, F3, F5 e F6) que não apresentaram resultados estatisticamente significativos, os dados adicionais, advindos de registros qualitativos (Diário de Campo e Questionários Inicial e Final), retrataram mudanças no repertório comportamental, que provavelmente somaram na significância do Escore Total desse instrumento, haja vista a elevação de todos os escores no pós-teste. Destaca-se: "lidar com críticas", "dizer não", "fazer pedidos", "ter autocontrole", "expor sentimentos negativos e positivos", "demonstrar carinho" e "ser assertivo" como as principais aquisições verbalizadas pelos participantes.

No início do THS realizado, os adolescentes apresentavam medo de punições e rejeições sofridas no cotidiano passado e, como consequência, aceitavam as críticas como "verdades absolutas" passando a se considerar como "fracassados" e apresentando sentimentos de incapacidade: "não consigo", "não sou capaz", "não sou tão bom quanto as demais pessoas". Diante desses sentimentos, passavam a ter comportamentos passivos, deixando de fazer atividades antes prazerosas, por medo de não atingirem as expectativas de outrem. Por outro lado, após a intervenção com o THS, conforme a racional-teórica defendida por Mota e Matos (2013), a melhora da qualidade das relações entre pares pôde elevar a autoestima, uma vez que, ao contar com fontes de apoio pessoal, os adolescentes puderam compartilhar suas dificuldades e aprimorar estratégias para enfrentá-las. Além disso, os adolescentes sentiram-se mais seguros e capazes de expressar suas ideias e sentimentos, estabelecendo relações mais positivas e empáticas, o que é socialmente reforçador, consequentemente, elevando a autoestima e consolidando a noção de que ela é mantida por contingências de reforçamento positivo de origem social (Meyer, 2011).

No final do THS realizado, os adolescentes avaliaram o programa de forma positiva. No entanto, embora os dados obtidos sejam satisfatórios, expuseram que o tempo destinado à duração do treinamento e às sessões foi pouco: "Ele deveria ser feito com mais pessoas e durar 01 (um) ano, isso ajudaria mais pessoas de idades variadas no seu dia-a-dia e na forma como veem o mundo." ( $p 5)$; "Eu achei incrível cada momento do treinamento, me faz querer mais." ( $p 6)$; "O treinamento foi essencial, poderia ter mais tempo." ( $p 4)$;

O treinamento foi realmente muito importante para mim, estou aprendendo a lidar comigo e com situações que com certeza vão acontecer no decorrer da minha vida e gostei muito de conhecer um pouco mais sobre os meus colegas de classe com quem eu não tinha tanta intimidade. Mais encontros, por favor! (p10). 
Considerando que adolescentes com baixa autoestima tendem a apresentar ideação suicida (Sábado et al., 2020), os seguintes resultados qualitativos revelam o potencial do THS em contribuir para a prevenção dessa ocorrência a partir do ambiente escolar: quanto à satisfação em viver, $60 \%(\mathrm{n}=6)$ da amostra não tinha esse sentimento no pré-teste; como exposto nas verbalizações: "Eu sinto que não mereço viver." ( $p 7)$; "Não sinto tanta vontade de viver, não tem nada no mundo que me faça querer viver." $(p 8)$ "; "Sinto que não mereço viver porque não acho que tenho muitas coisas que gosto e também me sinto como se os outros não gostassem de mim." ( $p 6)$. No pós-teste, todos os participantes passaram a relatar pensamentos positivos, que podem ser representados pelas verbalizações: "Vivendo eu posso aprender mais e alcançar meus objetivos." ( $p$ б); "Às vezes sinto vontade de explorar o mundo." ( $p 7)$; e “Acabar com a vida, não é a solução.” ( $p 8)$.

Desta maneira, o presente estudo ressalta a necessidade e a viabilidade de adolescentes com baixos níveis de autoestima serem assistidos em programas psicoeducativos que treinem suas habilidades sociais, a fim de promover estratégias de enfrentamento para lidar com as adversidades da fase de transição para a vida adulta. Sugere-se ainda, que futuras pesquisas contemplem essas variáveis a fim de fortalecer os resultados encontrados.

\section{Considerações Finais}

Os resultados do presente estudo mostraram que o THS realizado promoveu a elevação da autoestima de adolescentes, com implicações para a saúde preventiva junto ao referido público a partir do ambiente escolar. As habilidades sociais aprimoradas durante a intervenção possibilitaram que os participantes melhorassem suas relações interpessoais, principalmente no que tange às dificuldades apresentadas na emissão de comportamentos relacionados ao Autocontrole e à Assertividade.

As limitações deste estudo referem-se ao fato da amostra ser exclusivamente de uma escola privada e ter sido realizada em apenas um estado. Outro fator foi a ausência de avaliação de segmento para verificar se essas aquisições foram mantidas e/ou generalizadas para demais contextos. Levando em consideração tais limitações e visando o aprimoramento deste pré-experimento, recomenda-se que, em futuras pesquisas, a fim de fortalecer os achados deste estudo, sejam utilizadas amostras de alunos de escolas privadas e públicas, sejam feitas intervenções com estudantes de outras regiões do Brasil e seja realizada a avaliação de segmento.

A pesquisa contribuiu para demonstrar que o treinamento de habilidades sociais é um instrumento relevante para elevação da autoestima em adolescentes. Dessa forma, fica viável para futuros estudos focar essas habilidades específicas em novas intervenções que visem à promoção da saúde mental em adolescentes e realizando incrementos metodológicos para um maior controle experimental.

\section{Referências}

Campos, J. R., Del Prette, A., \& Del Prette, Z. A. P. (2014). Depressão na adolescência: habilidades sociais e variáveis sociodemográficas como fatores de risco/proteção. Estudos e Pesquisas em Psicologia, 14(2), 408-428. https://www.redalyc.org/pdf/4518/451844508003.pdf

Cardoso, J. K. S., Coelho, L. B., \& Martins, M. D. G. T. (2018). Crescer para saber: o treinamento de habilidades sociais e assertividade com adolescentes em âmbito escolar. Revista Eletrônica Estácio Papirus, 4(2), 214-231. http://periodicos.estacio.br/index.php/papirussantacatarina/article/view/4287/2102

Castro Sena, R. M., \& Maia, E. M. C. (2017). A utilização do constructo da autoestima nas pesquisas em saúde no Brasil: contribuições conceituais à prática clínica. Humanidades Médicas, 17(2), 383-395. http://www.humanidadesmedicas.sld.cu/index.php/hm/article/view/1043/pdf_6.

Coelho, C. C. de A., \& Dell'Aglio, D. D. (2019). Clima escolar e satisfação com a escola entre adolescentes de ensino médio. Revista Psicologia: Teoria e Prática, 21(1), 248-264. http://dx.doi.org/10.5935/1980-6906/psicologia.v21n1p265-281

Del Prette, Z. A. P., \& Del Prette, A. (2015). Inventário de Habilidades Sociais para adolescentes (IHSA-Del-Prette): Manual de aplicação, apuração e interpretação. Casa do Psicólogo.

Del Prette, Z. A., \& Del Prette, A. (2017). Competência social e habilidades sociais: Manual teórico-prático. Editora Vozes.

Feitosa, F. B. (2014). A depressão pela perspectiva biopsicossocial e a função protetora das habilidades sociais. Psicol. cienc. prof., 34(2), 488-499. https://doi.org/10.1590/1982-3703000992013. 
Feitosa, F. B., Del Prette, Z. A. P., \& Del Prette, A. (2012). Social skills and academic achievement: the mediating function of cognitive competence. Temas em Psicologia, 20(1), 61-70. https://www.redalyc.org/pdf/5137/513751439006.pdf.

Ferreira, F. R., Carvalho, M. A. G. D., \& Senem, C. J. (2016). Desenvolvendo habilidades sociais na escola: um relato de expe riência. Construção Psicopedagógica, 24(25), 84-98. http://pepsic.bvsalud.org/scielo.php?pid=S1415-69542016000100007\&script=sci_abstract\&tlng=en

Fogaça, F. F. S., Tatmatsu, D., Comodo, C. N., Del Prette, Z. A. P., \& Del Prette, A. (2019). O desenvolvimento de habilidades sociais na adolescência como ápice comportamental. Revista Brasileira de Terapia Comportamental e Cognitiva, 21(2), 217-231. https://doi.org/10.31505/rbtcc.v21i2.1162

Hutz, C. S. (2000). Adaptação da escala de autoestima de Rosenberg (Manuscrito não publicado). Universidade Federal do Rio Grande do Sul. Porto Alegre: [s.n.].

Hutz, C. S., \& Zanon, C. (2011). Revisão da adaptação, validação e normatização da escala de autoestima de Rosenberg. Avaliação Psicológica, 10(1), 41-49. http://pepsic.bvsalud.org/scielo.php?script=sci_arttext\&pid=S1677-04712011000100005

Jovarini, N. V., Leme, V. B. R., \& Correia-Zanini, M. R. G. (2018). Influence of social skills and stressors on academic achievement in the sixthgrade. Paidéia (Ribeirão Preto), 28, 1-9. https://doi.org/10.1590/1982-4327e2819

Leme, V. B. R., Del Prette, Z. A. P., \& Coimbra, S. (2015). Social skills, social support and well-being in adolescents of different family configurations. Paidéia (Ribeirão Preto), 25(60), 9-17. https://doi.org/10.1590/1982-43272560201503

Leme, V. B. R., Fernandes, L. D. M., Jovarini, N. V., Achkar, A. M. E., \& Del Prette, Z. A. P. (2016). Social skills program for adolescents in vulnerable social contexts. Psico-USF, 21(3), 595-608. https://doi.org/10.1590/1413-82712016210313

Meyer, D. D. S. T. (2011). A autoestima na perspectiva da análise do comportamento [Trabalho de Conclusão de Curso, Instituto Brasiliense de Análise do Comportamento]. IBAC. https://ibac.com.br/wp-content/uploads/2017/08/Monografia-Deise-Meyer.pdf.

Moreira, P. D. M. (2018). Violência e funcionamento psicológico na adolescência: o papel moderador do suporte social [Dissertação de Mestrado, Universidade Lusófona do Porto]. ReCiL. http://dspace.ulusofona.pt/handle/10437/8791.

Morowatisharifabad, M. A., Sharifzadeh, G. R., Miri, M., \& Dastjerdi, R. (2019). Investigating the life skills and self-esteem in teenage girls in Birjand, Iran. International Journal of Pediatrics, 7(6), 9623-9630. https://dx.doi.org/10.22038/ijp.2019.37425.3259

Mota, C. P., \& Matos, P. M. (2013). Peer attachment, coping, and self-esteem in institutionalized adolescents: The mediating role of social skills. European Journal of Psychology of Education, 28(1), 87-100. https://link.springer.com/article/10.1007/s10212-012-0103-z.

Paixão, R. F., Patias, N. D., \& Dell'Aglio, D. D. (2018). Autoestima e sintomas de transtornos mentais na adolescência: Variáveis associadas. Psicologia: Teoria e Pesquisa, 34, 1-8. https://doi.org/10.1590/0102.3772e34436

Pereira, A. S., Shitsuka, D. M., Parreira, F. J., \& Shitsuka, R. (2018). Metodologia da Pesquisa Científica. Santa Maria: UAB/NTE/UFSM. https://repositorio.ufsm.br/bitstream/handle/1/15824/Lic_Computacao_Metodologia-Pesquisa-Cientifica.pdf?sequence=1

Pereira-Guizzo, C. D. S., Prette, A. D., Prette, Z. A. P. D., \& Leme, V. B. R. (2018). Programa de habilidades sociais para adolescentes em preparação para o trabalho. Psicologia Escolar e Educacional, 22(3), 573-581. https://doi.org/10.1590/2175-35392018035449

Sábado, R. G, Domínguez, L. R., Iturbe, F. G., Hernández, M. S., \& Fonseca, L. L. (2020). Problemas de autoestima y depresión: Posibles factores de riesgo para la ocurrencia de un intento de suicidio. MULTIMED, 24. http://www.revmultimed.sld.cu/index.php/mtm/article/view/1860

Sbicigo, J. B., Bandeira, D. R., \& Dell'Aglio, D. D. (2010). Escala de autoestima de Rosenberg (EAR): Validade fatorial e con sistência interna. PsicoUSF, 15(3), 395-403. https://doi.org/10.1590/S1413-82712010000300012

Schultheisz, T. S. D. V., \& Aprile, M. R. (2015). Autoestima, conceitos correlatos e avaliação. Revista Equilíbrio Corporal e Saúde, 5(1), 36-48. https://revista.pgsskroton.com/index.php/reces/article/view/22

Seema, G. B., \& Kumar, G. V. (2018). Impact of social skills training on self-esteem among male and female adolescent students. Indian Journal of Positive Psychology, 9(1), 147-151. https://doi.org/10.15614/ijpp.v9i01.11761 